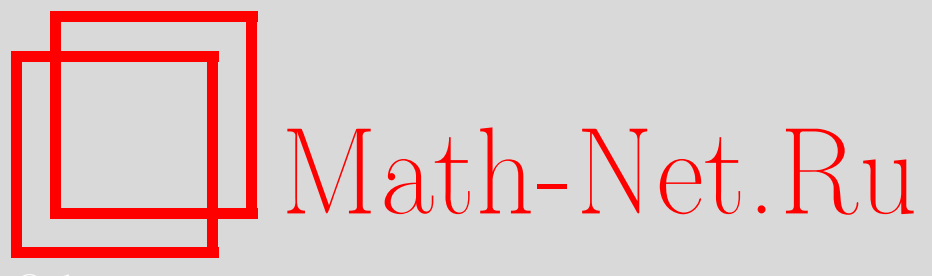

Д. Н. Карымов, О разложениях решетчатых распределений в свертки пуассоновских зарядов, Теория вероятн. и ее примен., 2004, том 49, выпуск 3, 589-596

DOI: https://doi.org/10.4213/tvp209

Использование Общероссийского математического портала Math-Net.Ru подразумевает, что вы прочитали и согласны с пользовательским соглашением

http://www . mathnet.ru/rus/agreement

Параметры загрузки:

IP : 54.198 .55 .26

26 апреля 2023 г., 13:29:44

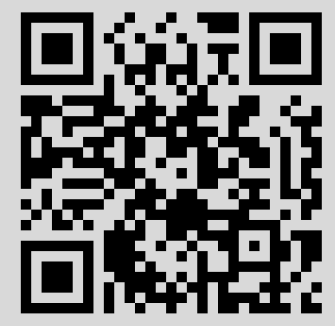


6. Vellaisamy P., Chaudhuri B. On compound Poisson approximation for sums of random variables. - Statist. Probab. Lett., 1999, v. 41, № 2, p. 179-189.

7. Wang Y. H. From Poisson to compound Poisson approximations. - Math. Sci., 1989, v. 14 , № 1 , p. $38-49$.

8. Wang Y. H. A compound Poisson convergence theorem. - Ann. Probab., 1991, v. 19, № 1, p. 452-455.

9. Абрамович М., Стиган И. (ред.). Справочник по специальным функциям с формулами, графиками и математическими таблицами. М.: Наука, 1979, 830 с.

Поступила в редакцию 15.III. 2004

(C) 2004 г.

КАРЫМОВ Д. Н.*

\title{
О РАЗЛОЖЕНИЯХ РЕШЕТЧАТЫХ РАСПРЕДЕЛЕНИЙ В СВЕРТКИ ПУАССОНОВСКИХ ЗАРЯДОВ ${ }^{1)}$
}

\begin{abstract}
В работе изложены некоторые результаты, связанные со свертками зарядов специального вида, называемых пуассоновскими. Указан простой способ получения асимптотических разложений в свертки пуассоновских зарядов, подходящий для широкого класса решетчатых распределений. Показана аналогия между такими разложениями и классическими для теории вероятностей разложениями Грама-Шарлье типа $A$ и $B$ и рядами Эджворта-Крамера.
\end{abstract}

Ключевые слова и фразы: пуассоновские заряды, свертки пуассоновских зарядов, асимптотические разложения, решетчатые случайные величины.

Целью данной работы является изложение некоторых результатов, связанных со свертками зарядов специального вида, называемых далее пуассоновскими. Будут рассмотрены некоторые теоретические аспекты, важные для понимания существа дела, но оставшиеся в тени в статьях других авторов. Так, например, будет указан простой способ получения асимптотических разложений в свертки пуассоновских зарядов, подходяший для широкого класса решетчатых распределений. Будет выявлена аналогия между такими разложениями и классическими для теории вероятностей разложениями Грама-Шарлье типа $A$ и $B$ и рядами Эджворта-Крамера. Также будет показано, что в случае решетчатых распределений использование сверток пуассоновских зарядов, во-первых, более естественно и во-вторых, в отличие от указанных выше разложений, дает монотонно убывающую погрешность аппроксимации при росте числа членов в разложении. Вопреки традиции, сравнение с работами других авторов будет проведено в середине работы.

Данная статья содержит только формулировки результатов, полученных автором, их полное доказательство будет опубликовано позднее.

Так как простым сжатием и сдвигом любая решетчатая случайная величина может быть переведена в целочисленную, то в дальнейшем рассматривается только этот частный случай решетчатых величин.

* Московский государственный университет им. М.В. Ломоносова, механикоматематический факультет, кафедра теории вероятностей, Ленинские горы, 119992 Москва, Россия; e-mail: dmitry.karimov@in-fusio.com

1) Работа выполнена при поддержке Российского фонда фундаментальных исследований (грант № 02-01-00600). 
Пусть $F(x)$ - функция распределения целочисленной случайной величины, $f(t)-$ соответствуюшая ей характеристическая функция. Очевидно, что $f(t)$ является $2 \pi-$ периодической непрерывной функцией. Разложение $f(t)$ в тригонометрический ряд имеет вид

$$
f(t)=\sum_{k=-\infty}^{\infty} p_{k} e^{i t k} .
$$

В правой части равенства стоит линейная комбинация функций $e^{i t k}$, которые являются характеристическими функциями распределений, сосредоточенных в одной точке. Функцию распределения с единичным скачком в точке $k$ обозначим $E_{k}(x)$. Тогда из разложения характеристической функции в тригонометрический ряд по экспонентам следует представление распределения в виде смеси вырожденных распределений:

$$
F(x)=\sum_{k=-\infty}^{\infty} p_{k} E_{k}(x) .
$$

Это самое простое и естественное «асимптотическое разложение» для целочисленных распределений. Как ни странно, разложение в свертку пуассоновских зарядов получается аналогично.

Пусть $f(t)$ не обращается в нуль. Это позволяет нам рассмотреть главную ветвь ее логарифма $\ln f(t)$. Если $f(t)$ не имеет витков вокруг нуля на отрезке $[-\pi, \pi]$, то $\ln f(t)$ также является $2 \pi$-периодической непрерывной функцией. Посмотрим, что получится, если разложить ее в тригонометрический ряд по экспонентам. Приведенную ниже цепочку рассуждений следует воспринимать лишь как демонстрацию основной идеи полученных в работе результатов, мы не будем указывать условия, необходимые для корректности следующих ниже равенств и переходов. Итак,

$$
\ln f(t)=\sum_{k=-\infty}^{\infty} \lambda_{k} e^{i t k},
$$

где $\lambda_{k}=(2 \pi)^{-1} \int_{-\pi}^{\pi} e^{-i t k} \ln f(t) d t$. Из равенства $f(-t)=\overline{f(t)}$ следует, что $\lambda_{k}$ действительные числа. Тогда

$$
\begin{aligned}
& f(t)=\exp \{\ln f(t)\}=\exp \left\{\sum_{k=-\infty}^{\infty} \lambda_{k} e^{i t k}\right\}, \quad f(0)=1=\exp \left\{\sum_{k=-\infty}^{\infty} \lambda_{k}\right\}, \\
& f(t)=\exp \left\{\sum_{k=-\infty}^{\infty} \lambda_{k}\left(e^{i t k}-1\right)\right\}=\prod_{k=-\infty}^{\infty} \exp \left\{\lambda_{k}\left(e^{i t k}-1\right)\right\} .
\end{aligned}
$$

Заметим, что в правых частях стоят функции, похожие на характеристические функции пуассоновских распределений, но в нашем случае величины $\lambda_{k}$ не обязательно являются положительными.

О п р е д е л е н и е. При $k \neq 0$ пгассоновским зарядом с действительным показателем $\lambda$ и параметром растяжения $k, k$ - целое, будем называть заряд, имеющий в точке $k l, l \geqslant 0$, вес, равный $\lambda^{l} e^{-\lambda} / l$ !. При $k=0$ пуассоновский заряд будем считать совпадающим с вырожденным распределением $E_{0}(x)$.

Функцию распределения пуассоновского заряда обозначим $P_{\lambda, k}(x)$.

Так как $\exp \left\{\lambda_{k}\left(e^{i t k}-1\right)\right\}$ является характеристической функцией заряда $P_{\lambda_{k}, k}(x)$, то разложение

$$
F(x)=\prod_{k=-\infty}^{\infty}{ }^{*} P_{\lambda_{k}, k}(x)
$$

получается как следствие разложения логарифма характеристической функции в тригонометрический ряд Фурье по экспонентам.

Заметим, что если характеристическая функция $f(t)$ имеет ненулевое число $L$ витков вокруг нуля, то мы можем рассмотреть распределение $E_{-L} * F(x)$, характеристическая функция которого равна $f(t) e^{-i t L}$ и не имеет витков вокруг нуля на 
отрезке $[-\pi, \pi]$. Тогда $E_{-L} * F(x)=\prod_{k=-\infty}^{\infty}{ }^{*} P_{\lambda_{k}, k}(x)$ и, следовательно,

$$
F(x)=\left(E_{L} * \prod_{k=-\infty}^{\infty}{ }^{*} P_{\lambda_{k}, k}\right)(x),
$$

где $\lambda_{k}=(2 \pi)^{-1} \int_{-\pi}^{\pi} e^{-i t k}(\ln f(t)-i t L) d t$.

Заметим, что для корректности всех указанных переходов достаточно условия, состоящего в том, что $f(t)$ не обрашается в нуль и распределение имеет первый момент.

Введенные выше пуассоновские заряды являются простейшими обобщениями пуассоновского распределения $P_{\lambda}(x)=\sum_{0 \leqslant k \leqslant x} \lambda^{k} e^{-\lambda} / k$ !. Мы просто отказались от условия $\lambda>0$ и ввели растяжение оси ординат в $k$ раз (при отрицательном $k$ это означает композицию растяжения и отражения). Отметим, что при $k=1$ и положительном показателе $\lambda$ пуассоновский заряд является обычным пуассоновским распределением. Такие заряды сохраняют многие свойства пуассоновского распределения. Tак, например, $P_{\lambda, k}(-\infty)=0, P_{\lambda, k}(\infty)=1$ и $P_{\lambda_{1}, k} * P_{\lambda_{2}, k}(x)=P_{\lambda_{1}+\lambda_{2}, k}(x)$.

Таким образом, разложение в свертку пуассоновских зарядов аналогично представлению распределения целочисленной величины в виде смеси вырожденных распределений. Продемонстрируем аналогию с классическими разложениями для случайных величин.

Более 100 лет назад Дж. Грам и К. Шарлье в своих работах [3] и [4] рассматривали логарифм характеристической функции биномиального распределения с параметрами $n$ и $p$. Разложив $\ln f(t)$ в ряд по степеням $t$, они получили разложение, которое теперь называют рядом Грама-Шарлье типа $A$ :

$$
S_{A}(x)=\phi(x)\left(1+\sum_{k=1}^{\infty} \frac{a_{k}}{k !} H_{k}(x)\right),
$$

где $\phi(x)=(2 \pi)^{-1 / 2} e^{-x^{2} / 2}-$ плотность нормального распределения, $H_{k}(x)=$ $(-1)^{k} e^{x^{2} / 2} \frac{d^{k}}{d x^{k}} e^{-x^{2} / 2}-$ многочлены Чебышева-Эрмита.

Впоследствии обобщение ряда Грама-Шарлье типа $A$ стало известно под названием ряда Эджворта--Крамера.

Разложив $\ln f(t)$ в ряд по степеням $p$, Грам и Шарлье получили представление, которое мы называем рядом Грама-Шарлье типа $B$ :

$$
S_{B}(x)=\Delta P_{\lambda}(x)+\sum_{k=1}^{\infty} \frac{b_{k}}{k !} \frac{d^{k} \Delta P_{\lambda}(x)}{d \lambda^{k}},
$$

где $\Delta P_{\lambda}(x)$ - величина скачка функции $P_{\lambda}$ в точке $x$.

Основной проблемой при использовании этих рядов является то обстоятельство, что частичные суммы ведут себя нерегулярно в том смысле, что сумма $m+1$ членов разложения может давать худшую аппроксимацию, чем сумма $m$ членов.

Выше показано, что разложение в свертку пуассоновских зарядов получается при разложении $\ln f(t)$ в ряд по степеням $e^{i t}$.

По-видимому, сейчас нельзя ответить на вопрос о том, почему Грам и Шарлье, рассматривая $2 \pi$-периодическую функцию, разложили ее в ряды по степеням $t$ и $p$, но не рассмотрели напрашивающегося разложения по степеням $e^{i t}$. И можно считать, что предпосылки для открытия разложений в свертки пуассоновских зарядов существовали еще около 100 лет назад, однако первые шаги в этой области были сделаны значительно позже.

По-видимому, пуассоновские заряды появились впервые в работах П. Корня и Э. Л. Пресмана в 1983 году. В 1986 году Ю. Круопис предложил использовать свертки пуассоновских зарядов для аппроксимации решетчатых распределений (см. [5] и [6]). Он пользовался методом, основанным на выравнивании первых $n$ моментов исходного распределения и свертки из $n$ пуассоновских зарядов. В отличие от описанного выше, его метод работает и в случае, когда $\ln f(t)$ обрашается в нуль, но он довольно сложен, так как для каждого $n$ требуется доказывать отдельную теорему. Так, Круопис ограничился рассмотрением аппроксимаций свертками не более трех зарядов, указав, что для большего числа зарядов вычисления становятся слишком громоздкими. Тем 
не менее, он показал, что свертка всего двух зарядов $P_{\lambda_{1}, 1} * P_{\lambda_{-1}, 1}(x)$ приближает биномиальное распределение с лучшей скоростью сходимости, чем при нормальном и пуассоновском приближении. Сравнивая результаты Круописа о приближении биномиального распределения сверткой $P_{\lambda_{1}, 1} * P_{\lambda_{2}, 2}(x)$ и полученные ниже оценки (см. теорему 4 при $N=2$ ), можно прийти к выводу, что оценки из теоремы 4 точнее лишь в довольно узкой зоне пуассоновской аппроксимации $n p^{2} \leqslant C$, тогда как результаты Круописа оказываются лучше в более широкой зоне нормальной аппроксимации. Впоследствии эти результаты были усилены А. Д. Бабуром и В. Чяканавичюсом, которые в своей работе [7] использовали метод Стейна-Чена. По-видимому, для свертки небольшого числа зарядов оценки, полученные этим методом, превосходят остальные по точности. Применение этого метода для большого числа зарядов затрудняется возрастающей сложностью доказательства и оценок.

Многие авторы (см. библиографию в [8] и [9]) в качестве дальнейшего развития результатов Круописа рассматривали обобщенный пуассоновский заряд вида $E_{0}(x)+\sum_{k=0}^{\infty} G^{* k}(x) / k$ !. В замечании 4 будет показано, что в тех же целях можно использовать обобщение более привычного вида.

В своей работе [10] Ш. Якшявичюс рассмотрел целочисленные распределения на положительной полуоси, удовлетворяющие условию $\Delta F(k) \leqslant\left(\frac{1}{2}-\varepsilon\right)^{k} \Delta F(0)$. Взяв производящую функцию распределения $\varphi(z)=\sum_{k=0}^{\infty} \Delta F(k) z^{k}$, он обозначил производные главной ветви ее логарифма в нуле $\sigma_{k}=\left.\frac{d^{k}}{d z^{k}} \ln \varphi(z)\right|_{z=0}$ и получил разложение $f(t)=\prod_{k=1}^{\infty} \exp \left\{\sigma_{k}\left(e^{i t k}-1\right) / k !\right\}$. По-видимому, автор не заметил связи между полученным разложением и работами Круописа, поэтому мы уделим этому некоторое внимание. Заметим, что при таких условиях на распределение $F(x)$ имеет место равенство

$$
\begin{aligned}
\lambda_{k} & =\frac{1}{2 \pi} \int_{-\pi}^{\pi} e^{-i t k} \ln f(t) d t=\frac{1}{2 \pi} \oint e^{-i t(k+1)} \ln \varphi\left(e^{i t}\right) d e^{i t} \\
& =\frac{1}{2 \pi} \oint_{|z|=1} z^{-k+1} \ln \varphi(z) d z=\left.\frac{1}{k !} \frac{d^{k}}{d z^{k}} \ln \varphi(z)\right|_{z=0}=\frac{\sigma_{k}}{k !} .
\end{aligned}
$$

Таким образом, Якшявичюс получил разложение $F(x)=\prod_{k=1}^{\infty}{ }^{*} P_{\lambda_{k}, k}(x)$. Очевидно, что для распределений, полученных сдвигом на целое число $l$, получается разложение $F(x)=\left(E_{L} * \prod_{k=1}^{\infty}{ }^{*} P_{\lambda_{k}, k}\right)(x)$. По-видимому, работа Якшявичюса является единственной на данный момент, в которой для частного случая получено исследуемое в нашей работе разложение. Также отметим, что определенные выше величины $\lambda_{k}$ являются коэффициентами ряда Лорана логарифма производящей функции распределения $\ln \varphi(z)=\sum_{k=-\infty}^{\infty} \lambda_{k} z^{k}$ и в более общем случае. Таким образом, Якшявичюс получил разложение, рассматривая ряд $\ln \varphi(z)$ в единичном круге, но его метод неприменим, если $\varphi(z)$ обрашается в нуль в этом круге. В данной статье разложение в свертку пуассоновских зарядов получено на основе разложения $\ln \varphi(z)$ в ряд по степеням $z$ на единичной окружности $z=e^{i t}$, и указанный метод не работает, если $\varphi(z)$ обращается в нуль на этой окружности. Вероятно, можно выбрать $r>0$ такое, что $\varphi(z)$ не обращается в нуль на окружности радиуса $r$, и построить разложение распределения в свертку пуассоновских зарядов, рассматривая степенной ряд $\varphi(z)$ на такой окружности.

Далее мы будем использовать следующие обозначения:

$$
\begin{aligned}
& \Delta H(y)=\lim _{x \downarrow y} H(x)-\lim _{x \uparrow y} H(x) \text { - величина скачка функции } H(x) \text { в точке } y, \\
& \aleph_{n}(F)=\left.(-i)^{n} \frac{d^{n} \ln f(t)}{d t^{n}}\right|_{t=0} \text { - семиинвариант порядка } n \\
& \text { функции распределения } F(x) .
\end{aligned}
$$

Теорема 1. Пусть $F(x)$ - Функиия распределения иелочисленной случайной величинь, $f(t)$ - ее характеристическая функиия, $f(t)$ не обрашается в нуль и делает $L$ витков вокруг нуля на отрезке $[-\pi, \pi]$. Пусть $\ln f(t)$ - главное значение логарифма $f(t)$,

$$
\lambda_{k}=\frac{1}{2 \pi} \int_{-\pi}^{\pi} e^{-i t k}(\ln f(t)-i t L) d t
$$


коэффичиенты Фурье тригонометрического ряда функиии $\ln f(t)$ - itL на отрезке $[-\pi, \pi]$.

Если ряд $\sum_{k=-\infty}^{\infty} \lambda_{k}$ абсолютно сходится, то распределение $F(x)$ можно представить в виде свертки

$$
F(x)=\left(E_{L} * \prod_{k=-\infty}^{\infty}{ }^{*} P_{\lambda_{k}, k}\right)(x)
$$

и для любого множества $A$ иелых иисел и любого $x$ выполнено неравенство

$$
\left|\Delta F(x)-\Delta\left(E_{L} * \prod_{k \in A}{ }^{*} P_{\lambda_{k}, k}\right)(x)\right| \leqslant \sqrt{\left|\sum_{\mathrm{a}} \lambda_{k}\right|^{2}+\sum_{\mathrm{a}} \lambda_{k}^{2}} S(F, A),
$$

здесь и далее $\sum_{\mathrm{a}}$ обозначает $\sum_{k \notin A \cup\{0\}}, a$

$$
S(F, A)=\exp \left\{2 \min \left(\sum_{\substack{k \in A \backslash\{0\} \\ \lambda_{k}<0}}\left|\lambda_{k}\right|, \sum_{\substack{k \notin A \cup\{0\} \\ \lambda_{k}>0}} \lambda_{k}\right)\right\} .
$$

Достаточным условием абсолютной сходимости ряда из коэффициентов Фурье некоторой функции является ее дифференцируемость (см. [1, с. 386]), поэтому справедливо следующее замечание.

3 а м е ч а н и е 1 . Если распределение $F(x)$ имеет первый момент, то в условиях теоремы ряд $\sum_{k=-\infty}^{\infty} \lambda_{k}$ абсолютно сходится.

3 а м е ч а н и е 2. Если ряд $\sum_{k=-\infty}^{\infty} \lambda_{k}$ абсолютно сходится, то определена бесконечная свертка $\prod_{k=-\infty}^{\infty}{ }^{*} P_{\lambda_{k}, k}(x)$ и имеют место формулы

$$
\prod_{k=-\infty}^{\infty}{ }^{*} P_{\lambda_{k}, k}(x)=\Delta\left(\prod_{k=-\infty}^{\infty}{ }^{*} P_{\lambda_{k}, k}\right)(x)=e^{\lambda_{0}} \sum_{\left\{l_{k}\right\}_{k \neq 0}: l_{k} \geqslant 0, \sum k l_{k}=x} \prod_{k \neq 0} \frac{\lambda_{k}^{l_{k}}}{l_{k} !} .
$$

Теорема 2. Если в условиях теоремы $1 F(x)$ имеет первый момент $u$ ряд $\sum_{k=-\infty}^{\infty} k \lambda_{k}$ абсолютно сходится, то для любого множества $A$ чельх чисел $и$ любого $0<a \leqslant \pi$ выполнено неравенство

$$
\begin{aligned}
& \max _{x}\left|F(x)-\left(E_{L} * \prod_{k \in A}{ }^{*} P_{\lambda_{k}, k}\right)(x)\right| \\
& \leqslant \frac{1}{2} S(F, A)\left(a \sum_{k \notin A}\left|k \lambda_{k}\right|+\frac{1}{\sqrt{2}} \sqrt{\frac{1}{a}-\frac{1}{\pi}} \sqrt{\left(\sum_{\mathrm{a}} \lambda_{k}\right)^{2}+\sum_{\mathrm{a}} \lambda_{k}^{2}}\right)
\end{aligned}
$$

и неравенство для расстояния полной вариации

$$
\begin{aligned}
& \operatorname{var}\left(F-E_{L} * \prod_{k \in A}{ }^{*} P_{\lambda_{k}, k}\right) \\
& \quad \leqslant S(F, A)\left(\left(1+\frac{\pi}{\sqrt{3}} \max _{[-\pi, \pi]}\left|\frac{f^{\prime}(t)}{f(t)}-i L\right|\right) \sqrt{\left(\sum_{\mathrm{a}} \lambda_{k}\right)^{2}+\sum_{\mathrm{a}} \lambda_{k}^{2}}+\frac{\pi}{\sqrt{3}} \sqrt{\sum_{\mathrm{a}} k^{2} \lambda_{k}^{2}}\right),
\end{aligned}
$$

а при $x \neq L$ справедлива и неравномерная оченка

$$
\begin{aligned}
& \left|\Delta F(x)-\Delta\left(E_{L} * \prod_{k \in A}{ }^{*} P_{\lambda_{k}, k}\right)(x)\right| \\
& \quad \leqslant \frac{1}{|x-L|} S(F, A)\left(\max _{\{-\pi, \pi]}\left|\frac{f^{\prime}(t)}{f(t)}-i L\right| \sqrt{\left(\sum_{\mathrm{a}} \lambda_{k}\right)^{2}+\sum_{\mathrm{a}} \lambda_{k}^{2}}+\sqrt{\sum_{\mathrm{a}} k^{2} \lambda_{k}^{2}}\right) .
\end{aligned}
$$

Eслип - натуральное число и ряд $\sum_{k=-\infty}^{\infty} k^{n} \lambda_{k}$ абсолютно сходится, то имеет место следующее равенство для разности между семиинвариантами конечной свертки и исходного распределения:

$$
\aleph_{n}(F)-\aleph_{n}\left(E_{L} * \prod_{k \in A}^{*} P_{\lambda_{k}, k}\right)=\sum_{\mathrm{a}} k^{n} \lambda_{k}
$$


3 а м е ч а н и е 3 . Если распределение $F(x)$ имеет второй момент, то в условиях теоремы ряд $\sum_{k=-\infty}^{\infty} k \lambda_{k}$ абсолютно сходится.

Следствие теоремы 2. Пусть $F(x)$ - функиия распределения иелочисленной случайной величины, имеющей второй момент. Пусть $f(t)-$ ее характеристическая функиия, $f(t)$ не обращается в нуль и делает $L$ витков вокруг нуля на отрезке $[-\pi, \pi]$. Пусть $\ln f(t)-$ главное значение логарифма $f(t)$, a

$$
\lambda_{k}=\frac{1}{2 \pi} \int_{-\pi}^{\pi} e^{-i t k}(\ln f(t)-i t L) d t
$$

- коэфФичиенть Фурье тригонометрического ряда функиии $\ln f(t)$ - itL на отрезке $[-\pi, \pi]$.

Тогда распределение $F(x)$ можно представить в виде свертки

$$
F(x)=\left(E_{L} * \prod_{k=-\infty}^{\infty}{ }^{*} P_{\lambda_{k}, k}\right)(x)
$$

и для любого множества $A$ чельх иисел выполнено

$$
\max _{x}\left|F(x)-\left(E_{L} * \prod_{k \in A}{ }^{*} P_{\lambda_{k}, k}\right)(x)\right| \leqslant \frac{\pi}{2} S(F, A) \sum_{k \notin A}\left|k \lambda_{k}\right| .
$$

Рассмотрим функцию распределения $F(x)$ целочисленной случайной величины, характеристическая функция которой $f(t)$ не обращается в нуль и делает $L$ витков вокруг нуля на отрезке $[-\pi, \pi]$. Теоремы 1 и 2 ставят в соответствие такому распределению функцию $\ln f(t)-i t L$ и свертку $\left(E_{L} * \prod_{k=-\infty}^{\infty}{ }^{*} P_{\lambda_{k}, k}\right)(x)$. Тогда распределению $\left(E_{M} * F\right)(x)$ будут соответствовать функция $\ln \left\{e^{i t M} f(t)\right\}-i t(M+L)$ и свертка $\left(E_{M+L} * \prod_{k=-\infty}^{\infty}{ }^{*} P_{\lambda_{k}, k}\right)(x)$. Распределению $F(x / m)$, полученному растяжением исходного распределения в $m$ раз, где $m$ - целое число, будут соответствовать $\ln f(m t)-i t m L$ и свертка $\left(E_{m L} * \prod_{k=-\infty}^{\infty}{ }^{*} P_{\lambda_{k}, m k}\right)(x)$. Таким образом, если мы пользуемся теоремами 1 или 2 , то наш метод «чувствует» сдвиги и растяжения исходного распределения.

Несколько приводимых ниже результатов посвящены вопросу снижения трудоемкости вычислений. Например, следуюшая теорема демонстрирует, что для нахождения коэффициентов $\lambda_{k}$ не всегда необходимо производить вычисление интегралов. В случае распределения с большим скачком в нуле справедливы более простые формулы, выражающие $\lambda_{k}$ через параметры распределения.

Теорема 3. Пусть $p_{k}=\Delta F(k), p_{0}>\frac{1}{2}$. Тогда коэффиииенть Фурье

$$
\lambda_{k}=\frac{1}{2 \pi} \int_{-\pi}^{\pi} e^{-i t k} \ln f(t) d t,
$$

где $\ln f(t)$ - главная ветвь логарифма, могут быть найдены по формулам

$$
\lambda_{k}= \begin{cases}\ln p_{0}+\sum_{n=1}^{\infty} \frac{(-1)^{n-1}}{n p_{0}^{n}} \sum{ }^{\prime} p_{k_{1}} \cdots p_{k_{n}}, & k=0 \\ \sum_{n=1}^{\infty} \frac{(-1)^{n-1}}{n p_{0}^{n}} \sum{ }^{\prime} p_{k_{1}} \cdots p_{k_{n}}, & k \neq 0\end{cases}
$$

$\sum^{\prime}$ означает суммирование по всем наборам $k_{1}, \ldots, k_{n}$ ненулевых чельх чисел таким, uто $k_{1}+\cdots+k_{n}=k$.

Следствие. Пусть $p_{k}=\Delta F(k), p_{k}=0$ при $k<0 u p_{0}>\frac{1}{2}$. Тогда коэфФициентьь

$$
\lambda_{k}=\frac{1}{2 \pi} \int_{-\pi}^{\pi} e^{-i t k} \ln f(t) d t
$$

где $\ln f(t)$ - главная ветвь логарифма, могут быть найдены по формулам.

$$
\lambda_{k}= \begin{cases}0, & k<0, \\ \ln p_{0}, & k=0, \\ \sum^{\prime \prime}(-1)^{\sum n_{l}-1}\left(\sum_{l=1}^{k} n_{l}-1\right) ! \prod_{l=0}^{k} \frac{1}{n_{l} !}\left(\frac{p_{l}}{p_{0}}\right)^{n_{l}}, & k>0,\end{cases}
$$


$\sum^{\prime \prime}$ означает суммирование по всем наборам $n_{1}, \ldots, n_{k}$ неотричательных челых чисел таким, ито $n_{1}+2 n_{2}+\cdots+k n_{k}=k$.

Отметим, что аналогичный результат может быть найден в работе Ш. Якшявичюса [10].

3 а м е ч а н и е 4. Пусть $\lambda_{k} \geqslant 0$ при $k \neq 0$ и $\sum_{k \neq 0} \lambda_{k}<\infty$. Возьмем произвольное число $\lambda \geqslant \sum_{k \neq 0} \lambda_{k}$ и обозначим $p_{k}=\lambda_{k} / \lambda$ при $k \neq 0$. Полученные величины неотрицательны и их сумма $\sum_{k \neq 0} p_{k}=\left(\sum_{k \neq 0} \lambda_{k}\right) / \lambda \leqslant 1$. Обозначим $p_{0}=1-\sum_{k \neq 0} p_{k}$ и возьмем функцию распределения целочисленной случайной величины с величинами скачков, равными $p_{k}: F(x)=\sum_{k \leqslant x} p_{k}$. Тогда $\prod_{k=-\infty}^{\infty}{ }^{*} P_{\lambda_{k}, k}(x)$ совпадает с обобщенным распределением Пуассона $e^{-\lambda} \sum_{k=0}^{\infty} \frac{\lambda^{k}}{k !} F^{* k}(x)$.

Обозначим

$$
B_{p, n}(x)= \begin{cases}0, & x<0, \\ \sum_{k=0}^{[x]} C_{n}^{k} p^{k}(1-p)^{n-k}, & 0 \leqslant x<n, \\ 1, & x \geqslant n,\end{cases}
$$

функцию распределения биномиального закона. Тогда при помощи теоремы 2 легко получить следующий результат.

Теорема 4. Пусть $0 \leqslant p<\frac{1}{2}, n u N-$ натуральные числа. Обозначим

$$
\begin{gathered}
\lambda_{k}=(-1)^{k-1} \frac{n}{k}\left(\frac{p}{1-p}\right)^{k} \quad \text { npu } \quad k>0 \\
S(N)=S(N, n, p)=\exp \left\{\frac{2 n p^{2}}{(N+1)(1-2 p)}\left(\frac{p}{1-p}\right)^{N-1}\right\} .
\end{gathered}
$$

Тогда имеют место: равенство распределений

локальная равномерная оченка

$$
B_{p, n}(x)=\prod_{k=1}^{\infty}{ }^{*} P_{\lambda_{k}, k}(x)
$$

$$
\max _{x}\left|\Delta B_{p, n}(x)-\Delta \prod_{k=1}^{N}{ }^{*} P_{\lambda_{k}, k}(x)\right| \leqslant \frac{1}{N+1} \sqrt{2+\frac{p^{2}}{1-2 p}} S(N) n\left(\frac{p}{1-p}\right)^{N+1},
$$

интегральная равномерная оченка

$$
\max _{x}\left|B_{p, n}(x)-\prod_{k=1}^{N}{ }^{*} P_{\lambda_{k}, k}(x)\right| \leqslant \frac{\pi}{2(1-2 p)} S(N) n\left(\frac{p}{1-p}\right)^{N+1},
$$

оченка для расстояния полной вариации

$$
\begin{aligned}
& \operatorname{var}\left|B_{p, n}-\prod_{k=1}^{N}{ }^{*} P_{\lambda_{k}, k}\right| \\
& \quad \leqslant S(N)\left(\frac{1}{N+1}\left(1+\frac{\pi}{\sqrt{3}} \frac{n p}{1-2 p}\right) \sqrt{2+\frac{p^{2}}{1-2 p}}+\frac{\pi}{\sqrt{3}} \frac{1-p}{\sqrt{1-2 p}}\right) n\left(\frac{p}{1-p}\right)^{N+1},
\end{aligned}
$$

при $x \neq 0$ локальная неравномерная оченка

$$
\begin{aligned}
& \left|\Delta B_{p, n}(x)-\Delta \prod_{k=1}^{N}{ }^{*} P_{\lambda_{k}, k}(x)\right| \\
& \quad \leqslant \frac{1}{|x|} S(N)\left(\frac{n p}{(N+1)(1-2 p)} \sqrt{2+\frac{p^{2}}{1-2 p}+\frac{1-p}{\sqrt{1-2 p}}}\right) n\left(\frac{p}{1-p}\right)^{N+1}
\end{aligned}
$$

и для любого натурального $m$ равенство для разности семичнвариантов

$$
\aleph_{m}\left(B_{p, n}\right)-\aleph_{m}\left(\prod_{k=1}^{N}{ }^{*} P_{\lambda_{k}, k}\right)=n \sum_{k=N+1}^{\infty}(-1)^{k-1} k^{m}\left(\frac{p}{1-p}\right)^{k} .
$$

Автор благодарит В.В. Сенатова за постановку задачи и всестороннюю поддержку. 


\section{СПИСОК ЛИТЕРАТУРЫ}

1. Зигмунд А. Тригонометрические ряды. Т. 1. М.: Мир, 1965, 615 с.

2. Прохоров Ю.В. Асимптотическое поведение биномиального распределения. Успехи матем. наук, 1953, т. 8, в. 3, с. 135-142.

3. Gram J. P. Über die Entwickelung reeller Functionen in Reihen mittelst der Methode der kleinsten Quadrate. - J. Reine Angew. Math., 1883, B. 94, S. 41-73.

4. Charlier C. V. L. Contributions to the mathematical theory of statistics. 5. - Arkiv. Mat. Astr. Fys., 1914, B. 9, H. 3-4, № 25, 17 S.

5. Круопис Ю. Точность аппроксимации обобщенного биномиального распределения свертками пуассоновских мер. - Литов. матем. сб., 1986, т. 26, в. 1, с. 53-69.

6. Круопис Ю. Аппроксимации распределений сумм решетчатых случайных величин. I, II. - Литов. матем. сб., 1986, т. 26, в. 3, с. 455-467; в. 4, с. 692-704.

7. Barbour A.D., Cekanavičius $V$. Total variation asymptotics for sums of independent integer random variables. - Ann. Probab., 2002, v. 30, № 2, p. 509-545.

8. Barbour A.D., Chryssaphinou O. Compound Poisson approximation: a user's guide. - Ann. Appl. Probab., 2001, v. 11, № 3, p. 964-1002.

9. Чяканавичюс $B$. Об обобщенных пуассоновских аппроксимациях при моментных ограничениях. - Теория вероятн. и ее примен., 1999, т. 44, в. 1, с. 74-86.

10. Якшявичюс ШI. О некотором способе разложения вероятностей решетчатых случайных величин. - Теория вероятн. и ее примен., 1997, т. 42, в. 2, с. 294-307.

Поступила в редакцию 16.IV.2004

(C) 2004 г.

\section{ФЕЛЬДМАН Г. М.*, ГРАЧИК П..* \\ К ТЕОРЕМЕ СКИТОВИЧА-ДАРМУА ДЛЯ ДИСКРЕТНЫХ АБЕЛЕВЫХ ГРУПП}

Доказана следующая теорема. Пусть $X-$ счетная дискретная абелева группа, $\operatorname{Aut}(X)$ - группа автоморфизмов группы $X, \xi_{1}, \xi_{2}-$ независимые случайные величины со значениями в $X$ и с распределениями $\mu_{1}, \mu_{2}$. Пусть $\alpha_{j}, \beta_{j} \in \operatorname{Aut}(X)$. Тогда из независимости линейных статистик $L_{1}=\alpha_{1} \xi_{1}+\alpha_{2} \xi_{2}$ и $L_{2}=\beta_{1} \xi_{1}+\beta_{2} \xi_{2}$ следует, что $\mu_{1}, \mu_{2}-$ идемпотентные распределения.

Ключевые слова и фразы: независимые линейные статистики, дискретные абелевы группы, теорема Скитовича-Дармуа.

Пусть $X$ - локально компактная сепарабельная абелева метрическая группа, $\operatorname{Aut}(X)$ - группа топологических автоморфизмов группы $X, \xi_{j}, j=1, \ldots, n, n \geqslant 2,-$ независимые случайные величины со значениями в группе $X$ и с распределениями $\mu_{j}$. Рассмотрим следуюшую задачу. Для каких групп $X$ из независимости линейных статистик $L_{1}=\alpha_{1} \xi_{1}+\cdots+\alpha_{n} \xi_{n}$ и $L_{2}=\beta_{1} \xi_{1}+\cdots+\beta_{n} \xi_{n}, n \geqslant 2$, где $\alpha_{j}, \beta_{j} \in$ $\operatorname{Aut}(X)$, следует, что все распределения $\mu_{j}$ - гауссовские с точностью до сдвигов на

* ФТИНТ НАН Украины, пр. Ленина, 47, Харьков, 103, 61103 УКРАИНА; е-mail: feldman@ilt.kharkov.ua

** Université d'Angers, Departement de Mathématiques, 2, boulevard Lavoisier, 49045 Angers Cedex 01, France e-mail: graczyk@tonton.univ-angers.fr

1) Первый автор частично поддержан грантом Ministère des Affaires Etrangères, France; второй автор поддержан the European Commision (TMR 1998-2006 Network Harmonic Analysis). 